\title{
Bronchial morphological changes are associated with postoperative intractable cough after right upper lobectomy in lung cancer patients
}

\author{
Xue-Fang Lu ${ }^{1 \#}$, Xin-Ping Min ${ }^{2 \#}$, Biao Lu ${ }^{3}$, Guo-Hua Fan ${ }^{3}$, Tie-Yuan Zhu ${ }^{3}$ \\ ${ }^{1}$ Department of Radiology, Renmin Hospital of Wuhan University, Wuhan, China; ${ }^{2}$ Department of Cardiovascular Surgery, Renmin Hospital of \\ Wuhan University, Wuhan, China; ${ }^{3}$ Department of Thoracic Surgery, Renmin Hospital of Wuhan University, Wuhan, China
}

Contributions: (I) Conception and design: TY Zhu; (II) Administrative support: TY Zhu; (III) Provision of study materials or patients: XF Lu, XP Min, GH Fan; (IV) Collection and assembly of data: XF Lu, XP Min, B Lu, GH Fan; (V) Data analysis and interpretation: XF Lu, XP Min, TY Zhu; (VI) Manuscript writing: All authors; (VII) Final approval of manuscript: All authors.

${ }^{\#}$ These authors contributed equally to this work.

Correspondence to: Dr. Tie-Yuan Zhu. Department of Thoracic Surgery, Renmin Hospital of Wuhan University, No. 238 Jiefang Road, Wuchang District, Wuhan 430060, China. Email: michelmeis@126.com.

Background: To date, postoperative intractable cough (PIC) has not received adequate attention, and the complex perioperative factors when performing pulmonary resection often prevent researchers from addressing this issue. This study aimed to investigate the clinicopathological and radiographic indicators related to PIC in lung cancer patients.

Methods: In all, 112 patients who had had right upper lobectomy for primary lung cancer from January 2019 to December 2020 were retrospectively reviewed. We collected data via the electronic medical database of our department. Bronchial morphological features were investigated comprehensively via threedimensional chest computer tomography reconstruction images.

Results: During outpatient follow-up visits, 41 (36.6\%) patients complained about persistent dry cough after surgery. Compared with the non-cough group, patients in the refractory cough group showed significant differences in smoking history, right upper lobe stump length, changes of right bronchus intermedius (RBI) diameter, changes of right lower lobe (RLL) basal bronchus diameter, changes of RBI/ RLL bronchial angle, and bronchial kink. However, according to multivariable regression analysis, stump length, bronchial kink, and diameter change of the right lower lobe basal bronchus were independently associated with postoperative refractory cough. A nebulization drug was prescribed for the 41 patients diagnosed with PIC, and 33 (80.5\%) patients had improved by the next visit.

Conclusions: After right upper lobectomy, the morphology of the remaining bronchial tree in the residual lung changed significantly. The bronchial morphological alterations were independent risk factors for PIC.

Keywords: Postoperative cough; risk factor; bronchial morphology; right upper lobectomy; lung cancer

Submitted Apr 05, 2021. Accepted for publication Jun 11, 2021.

doi: 10.21037/qims-21-368

View this article at: https://dx.doi.org/10.21037/qims-21-368 


\section{Introduction}

Lobectomy and systematic lymph node dissection are standardized treatments for invasive lung cancer (1). Due to improved recovery after surgery, the average length of stay for lobectomy has decreased to 1 to 2 days postoperatively. New-onset, persistent, nonproductive cough after pulmonary resection is not uncommon. The reported incidence in the literature is as high as $50 \%$ following pulmonary resection (2). Refractory cough is painful. Once it begins, most patients are unwilling to leave the hospital and cannot typically to return to their work and life.

To date, postoperative intractable cough (PIC) has not yet received adequate attention. The often complex perioperative factors when performing pulmonary resection prevent researchers from addressing the issue of PIC. A range of reasons are assumed to account for this, including airway infection, asthma, end-bronchial sutures, lymph node dissection, gastrointestinal reflux, postoperative physical deconditioning, and bronchial kink $(3,4)$. Following right upper lobectomy (RUL), the remaining trachea and lungs need to adapt to the new conditions. The upward movement of the middle and lower lobes are physiological, but sometimes bronchial remodeling induces excessive deformation leading to symptoms and functional problems after lung resection (5).

In this study, we focused on patients with lung cancer who underwent right upper lobectomy to investigate the association between PIC and clinicopathological features and radiographic indicators.

\section{Methods}

\section{Participants}

In all, 112 patients who underwent RUL for primary lung cancer at our institute from January 2019 to December 2020 were retrospectively reviewed. We collected data via the electronic medical database of our department. The inclusion criteria were as follows: (I) non-small cell lung cancer (NSCLC) patients (II) tumor(s) located in the right upper lobe, (III) history of RUL and systematic mediastinal lymph node dissection, (IV) no obvious preoperative cough, (V) complete (R0) resection, (VI) no neoadjuvant antitumor treatment, and (VII) no recent neck surgery. The exclusion criteria were as follows: (I) combined surgical procedures, such as a wedge resection in other lobe; (II) major respiratory complications, such as postoperative pneumonia or respiratory failure; and (III) an incomplete hospital record. The flowchart of patient selection for the study is shown in Figure 1. The study was conducted in accordance with the Declaration of Helsinki (as revised in 2013). This study was approved by the Renmin Hospital of Wuhan University Review Board for Clinical Research. The informed consent requirement was waived because of the retrospective study design.

\section{Operative techniques}

Both video-assisted thoracoscopic surgery (VATS) and thoracotomy were used for RUL. The typical VATS procedures in our department are conducted via 2 ports. For central type lung carcinoma or giant tumors (more than $5 \mathrm{~cm}$ in diameter), a right anterolateral thoracotomy is preferred. We used a single-direction approach to perform the RUL. The bronchi and vessels were resected using endoscopic staplers (Articulating Endoscopic Linear Cutter PSE45A; Ethicon Endo-Surgery, LLC; Johnson \& Johnson, New Brunswick, NJ, USA). In some patients, surgeons sutured the bronchial stumps (4-0 Prolene, Ethicon EndoSurgery) based on the surgeon's discretion. The $2 \mathrm{R}, 4 \mathrm{R}$, $7,8,9$, and 10 groups of lymph nodes were sampled and dissected. Before closing the incision, a $26 \mathrm{~F}$ chest tube was placed towards the thoracic apex, and a $19 \mathrm{~F}$ fine drainage tube was placed towards the costodiaphragmatic sinus.

\section{Postoperative management}

Patients received antibiotics and Mucosolvan therapies for about 3 to 5 days. The chest tube was removed if the following conditions were met: (I) good expansion of remaining lung, (II) no obvious pleural effusion, (III) no air leakage, and (IV) plasma drainage fluid of less than $200 \mathrm{~mL} / \mathrm{d}$.

For patients with dry cough after surgery, our protocol was initial administration of symptomatic therapies, including methoxamine and pectoral syrups. For patients who failed to respond to the above 2 drugs, A neubilization drug (SYMBICORT ${ }^{\circledR}$, Budesonide and formoterol powder for inhalation; AstraZeneca AB, Stockholm, Sweden) was prescribed.

\section{Outpatient records}

Patients were followed up in our outpatient center after discharge. Routine visits were scheduled at 2 weeks, 1 month, and then every 3 months during the first year and 


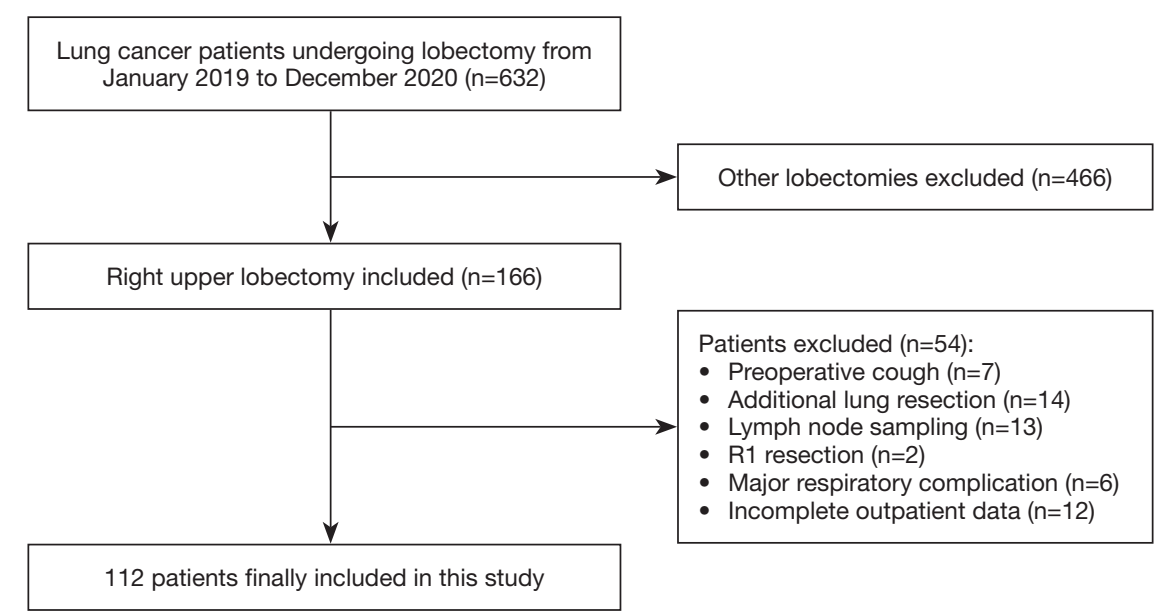

Figure 1 Flowchart of patient selection for inclusion in the study.

every 6 months thereafter. Outpatient medical records were checked and collected, including symptoms, radiological examinations, and medications.

The definition of postoperative intractable cough in this study was (I) new-onset dry cough after lung resection, (II) clear etiology with postnasal drip syndrome (PNDS) being excluded, (II) cough lasting more than 3 weeks after surgery, and (IV) normal blood tests and chest radiographs.

\section{X-ray-based measurement}

Chest X-ray (posterior-anterior position) was used to evaluate the dead space of the right thoracic cavity and whether there was obvious pleural effusion. The ratio of dead space was measured according to the method introduced by Matsuoka et al. (6).

\section{Three-dimensional (3D) chest computer tomography reconstruction-based measurement}

The 1-month follow-up visit included a routine thin-slice chest computer tomography (CT) examination. Threedimensional reconstructions of $\mathrm{CT}$ s for each patient were conducted by two authors (XFL and XPM) using the Materialise Mimics V. 20.0 software (Materialise LLC, Leuven, Belgium) for both preoperative and postoperative images. The following indicators of bronchial morphological changes were investigated: (I) changes of bronchial diameters at different levels, (II) changes of the angles between different bronchi, (III) bronchial kink, and (IV) the lengths of the upper lobe bronchial stumps.
Measurement of the bronchial diameters and angles was performed manually for the coronal and transverse planes of the 3D reconstruction images (Figure 2). The final values were the averages of the 2 data collectors. If the differences were too substantial, a third researcher was consulted. The definition of bronchial kink was the presence of bronchial angulation resulting in severe airway narrowing as reported by Ueda et al. (7) and as shown in Figure 3. The postoperative major (a) and minor (b) diameters of the bronchi and corresponding preoperative diameters (A and $\mathrm{B}$ ) were measured in $3 \mathrm{D}$ reconstructive images. Severe bronchial narrowing was defined as more than $75 \%$ (narrowing index $=1-\mathrm{ab} / \mathrm{AB}$ ).

\section{Statistical analysis}

Data are presented as either mean \pm standard deviation or percentages. The Student's $t$ test and Mann-Whitney test were used for continuous and discrete variables. The chisquare test was used for categorical variables. Statistical significance was set at a $\mathrm{P}$ value less than 0.05 . Risk factors for intractable cough were investigated using logistic regression model analysis. The SPSS version 22.0 (IBM Corp., Armonk, NY, USA) was used.

\section{Results}

\section{Patient characteristics}

There were $66(58.9 \%)$ males with a median age of 61 years. Nearly half of all patients $(46.4 \%)$ had a history of smoking; however, only $9.8 \%$ of patients had chronic 

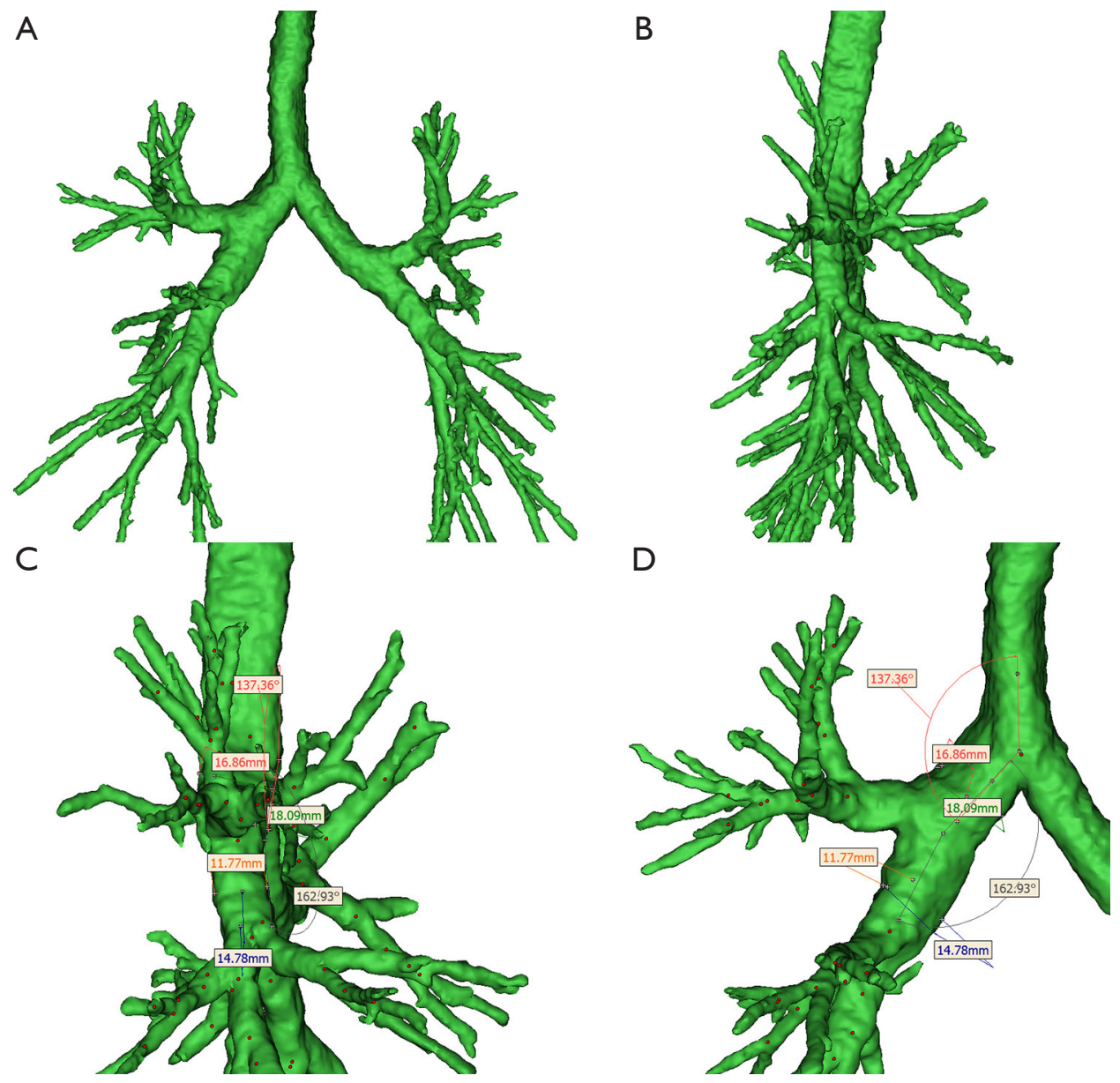

Figure 2 Three-dimensional (3D) chest CT reconstruction images on the coronal (A)/transverse (B) planes and examples of measurement of the bronchial diameters and angles on the coronal (C)/transverse (D) planes.

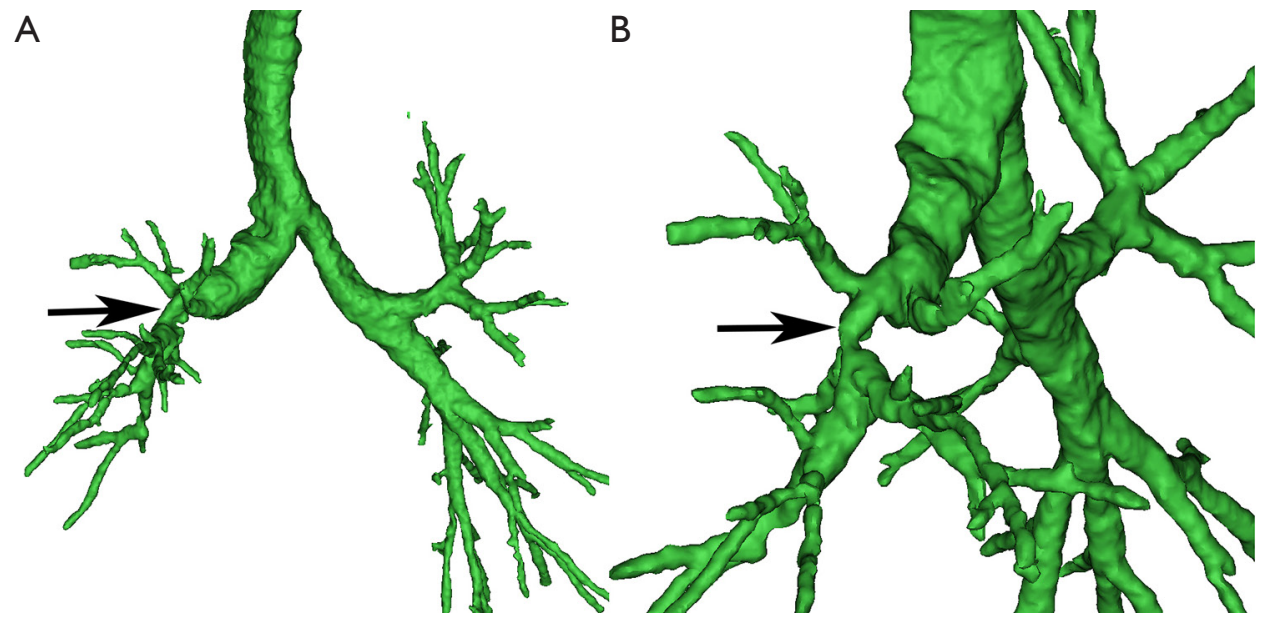

Figure 3 Three-dimensional bronchographic images showing postoperative bronchial kink (arrow; Figure 2 and Figure 3 are from the same patient). 
Table 1 Preoperative and perioperative patient characteristics

\begin{tabular}{|c|c|}
\hline Characteristics & Data \\
\hline No. of patients & 112 \\
\hline Age (years) & $61.2 \pm 9.8$ \\
\hline Male & $66(58.9)$ \\
\hline Smoking history & $52(46.4)$ \\
\hline COPD & $11(9.8)$ \\
\hline \multicolumn{2}{|l|}{ Surgical approach } \\
\hline VATS & $13(11.6)$ \\
\hline Thoracotomy & $99(88.4)$ \\
\hline Reinforcement of bronchial stump & $14(12.5)$ \\
\hline \multicolumn{2}{|l|}{ Pathology } \\
\hline Adenocarcinoma & $104(92.9)$ \\
\hline Squamous carcinoma & $8(7.1)$ \\
\hline \multicolumn{2}{|l|}{ T stage } \\
\hline 1 & $98(87.5)$ \\
\hline 2 & $11(9.8)$ \\
\hline 3 & $3(2.7)$ \\
\hline 4 & 0 \\
\hline \multicolumn{2}{|l|}{$\mathrm{N}$ stage } \\
\hline 0 & $102(91.1)$ \\
\hline 1 & $7(6.3)$ \\
\hline 2 & $3(2.7)$ \\
\hline
\end{tabular}

Data are presented as mean \pm standard deviation or percentage (\%). COPD, chronic obstructive pulmonary disease; VATS, video-assisted thoracoscopic surgery.

obstructive pulmonary disease (COPD). The majority (88.4\%) of patients underwent right upper lobectomy via a thoracoscopy approach. The stumps of the right upper bronchus were sutured using 4-0 Prolene in 14 (12.5\%) patients. The pathological findings revealed that $92.9 \%$ patients had an adenocarcinoma. With regard to the tumor, nodes, and metastases (TNM) classification, $87.5 \%$ were T1, 9.8\% T2, and $2.7 \% \mathrm{~T} 3$, while $91.1 \%$ were N0, $6.3 \%$ $\mathrm{N} 1$, and $2.7 \% \mathrm{~N} 2$. The preoperative and perioperative characteristics of patients are summarized in Table 1.

\section{Outpatient outcome of intractable cough}

During the first postoperative visit, half of all patients exhibited a cough of different degrees of severity. One month after surgery, $41(36.6 \%)$ patients complained of persistent dry cough during their visits.

\section{Bronchial morphological changes in radiology}

Based on the $3 \mathrm{D}$ reconstruction images, we found that the average lumen diameters of all the bronchi decreased significantly after surgery. The bronchial angle, changes in the trachea and right principal bronchus (trachea/RPB) angle increased slightly; however, other angles between different bronchi, such as the right principal bronchus/right bronchus intermedius (RPB/RBI) angle and right bronchus intermedius/right middle lobe (RBI/RML) bronchus angle, were all significantly reduced. The RUL stumps ranged from 1.6 to $10.1 \mathrm{~mm}$ with a mean value of $3.1 \mathrm{~mm}$. The detailed data of bronchial morphological changes are summarized in Table 2. In addition, it is worth mentioning that $27(24.1 \%)$ patients demonstrated bronchial kink in $3 \mathrm{D}$ reconstruction images. Tortile bronchi were found in 14 patients in the RLL basal bronchus, 6 in the RLL dorsal bronchus, and 7 in the RML bronchus.

\section{Univariate and multivariate analyses of risk factors}

Variables that likely influenced postoperative intractable cough were analyzed between groups (Table 3). Compared with the non-cough group, patients in the refractory cough group showed significant differences in smoking history, RUL stump length, changes of RBI diameter, changes of RLL basal bronchus diameter, changes of RBI/RLL bronchial angle, and bronchial kink. The above indicators were then entered into a multivariable regression model, and RUL stump length, bronchial kink, and diameter change of RLL basal bronchus were independently associated with postoperative refractory cough (Table 4).

\section{Potential benefit of nebulization drugs}

When the 41 patients who did not respond to common antitussive drugs complained of refractory cough during the 1 -month visit, the nebulization drug Symbicort was offered. During the next visit, cough was alleviated in 33 (80.5\%) patients according to medical records. We analyzed the clinical demographics of all 41 PIC patients. Unfortunately, no indicators showed a statistically significant difference between the cough improvement and no-improvement groups, probably due to the relatively small number of cases. 
Table 2 Radiological findings of bronchial morphological changes after surgery

\begin{tabular}{|c|c|c|c|}
\hline Morphological indicators & Preoperative & Postoperative & $P$ value \\
\hline \multicolumn{4}{|l|}{ Lumen diameter (mm) } \\
\hline RPB (major axis) & $13.7 \pm 2.9$ & $12.0 \pm 2.0$ & $<0.001$ \\
\hline RPB (minor axis) & $11.8 \pm 2.6$ & $9.5 \pm 1.4$ & $<0.001$ \\
\hline RBI (minor axis, cm) & $8.6 \pm 2.3$ & $8.3 \pm 1.7$ & 0.015 \\
\hline RML bronchus (major axis) & $6.5 \pm 0.6$ & $5.5 \pm 0.8$ & $<0.001$ \\
\hline RML bronchus (minor axis) & $5.8 \pm 0.6$ & $5.1 \pm 0.8$ & $<0.001$ \\
\hline RLL bronchus (major axis) & $8.4 \pm 1.6$ & $8.0 \pm 1.2$ & 0.001 \\
\hline RLL dorsal bronchus (minor axis) & $5.6 \pm 1.2$ & $4.9 \pm 1.4$ & $<0.001$ \\
\hline RLL basal bronchus (major axis) & $5.0 \pm 1.1$ & $4.4 \pm 0.9$ & $<0.001$ \\
\hline RLL basal bronchus (minor axis) & $4.2 \pm 0.9$ & $3.9 \pm 0.8$ & $<0.001$ \\
\hline \multicolumn{4}{|l|}{ Bronchial angle $\left(^{\circ}\right)$} \\
\hline Trachea/RPB & $136.5 \pm 3.6$ & $139.0 \pm 4.7$ & $<0.001$ \\
\hline $\mathrm{RPB} / \mathrm{RBI}$ & $167.1 \pm 4.7$ & $162.0 \pm 4.4$ & $<0.001$ \\
\hline RBI/RML bronchus & $135.6 \pm 4.2$ & $126.9 \pm 6.2$ & $<0.001$ \\
\hline RBI/RLL bronchus & $161.7 \pm 5.9$ & $155.2 \pm 6.2$ & $<0.001$ \\
\hline
\end{tabular}

Data are presented as mean \pm standard deviation. RUL, right upper lobe; N/A, not available; RPB, right principal bronchus; RBI, right bronchus intermedius; RML, right middle lobe; RLL, right lower lobe.

Table 3 Variables influencing postoperative intractable cough

\begin{tabular}{lccc}
\hline & \multicolumn{2}{c}{ Postoperative intractable cough } & No $(\mathrm{n}=71)$ \\
\hline Age (years) & Yes $(\mathrm{n}=41)$ & $54.4 \pm 9.4$ & 0.711 \\
Female & $53.1 \pm 11.4$ & $34(47.9)$ & 0.073 \\
Smoking history & $12(29.3)$ & $27(38.0)$ & 0.019 \\
COPD & $25(61.0)$ & $6(8.5)$ & 0.743 \\
VATS & $5(12.2)$ & $62(87.3)$ & 0.765 \\
Reinforcement of stump & $37(90.2)$ & $9(12.7)$ & 1.000 \\
Adenocarcinoma & $5(12.2)$ & $67(94.4)$ & 0.461 \\
\hline
\end{tabular}

Table 3 (continued) 
Table 3 (continued)

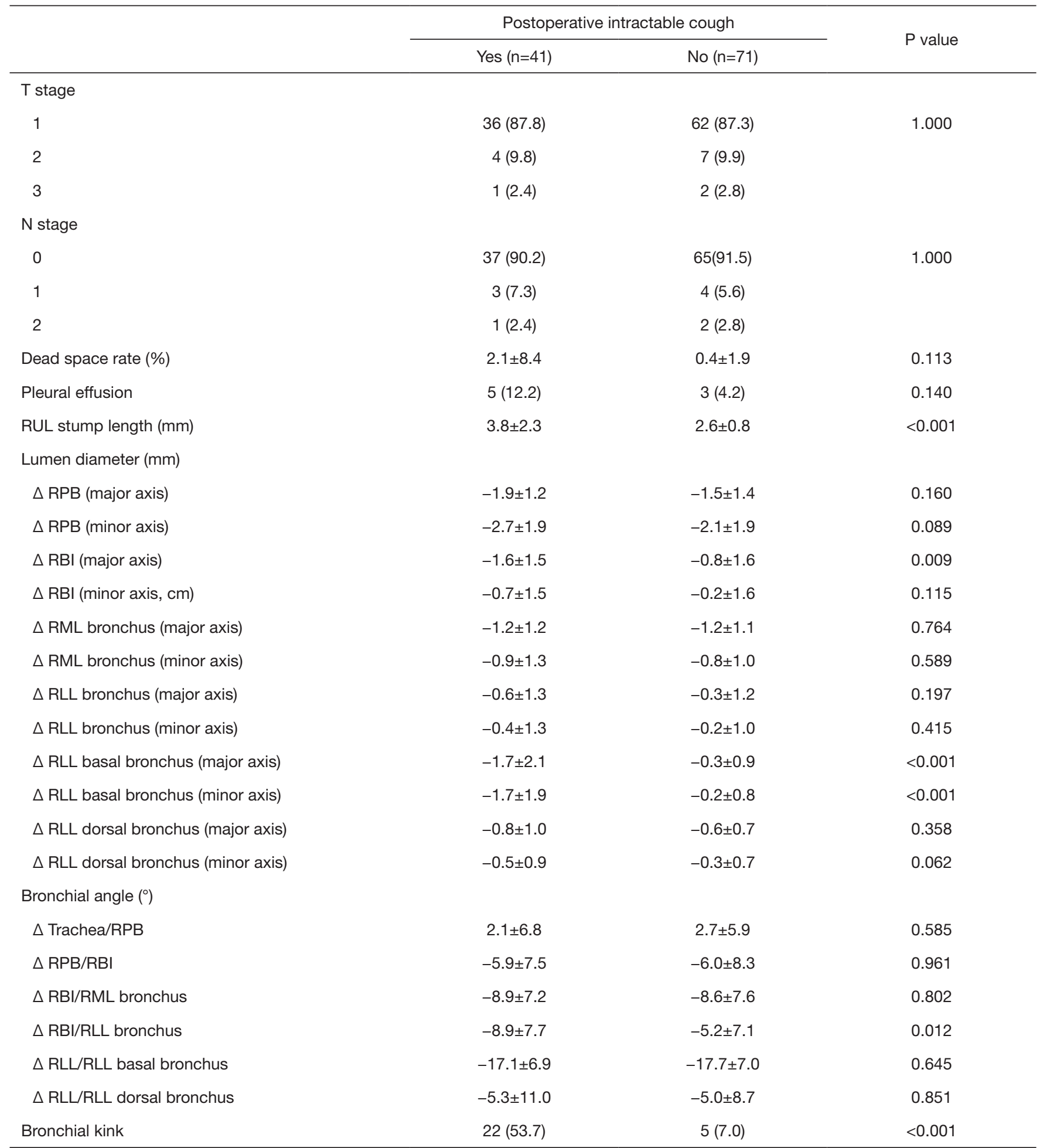

Data are presented as mean \pm standard deviation or percentage (\%). " $\triangle$ " represents the changes of bronchial diameter/angle after surgery. COPD, chronic obstructive pulmonary disease; VATS, video-assisted thoracoscopic surgery; RUL, right upper lobe; RPB, right principal bronchus; RBI, right bronchus intermedius; RML, right middle lobe; RLL, right lower lobe. 
Table 4 Independent risk factors of postoperative intractable cough

\begin{tabular}{lccc}
\hline Indicators & OR & $95 \% \mathrm{Cl}$ & $\mathrm{P}$ value \\
\hline Smoking history & 2.345 & $0.609-9.036$ & 0.215 \\
RUL stump & 1.904 & $1.185-3.058$ & 0.008 \\
Bronchial kink & 20.855 & $4.718-92.184$ & $<.001$ \\
$\Delta$ RBI (major axis) & 0.693 & $0.435-1.103$ & 0.122 \\
$\Delta$ RLL basal bronchus (major axis) & 0.408 & $0.170-0.982$ & 0.045 \\
$\Delta$ RBI/RML bronchus & 1.046 & $0.968-1.130$ & 0.256 \\
\hline
\end{tabular}

$\mathrm{RUL}$, right upper lobe; $\mathrm{RBI}$, right bronchus intermedius; RLL, right lower lobe; RML, right middle lobe; OR, odds ratio. " $\triangle$ " represents the changes of bronchial diameter/angle after surgery.

\section{Discussion}

Given the changes in the disease spectrum, the proportion of asymptomatic patients with early lung cancer has increased gradually in clinical practice (8). The fast-track recovery management of video-assisted thoracoscopic surgery (VATS) for lung cancer minimizes injury to and impact of the surgery on patients when a lobectomy is performed (9). Single-port VATS and multimodal analgesia relieves the refractory pain after surgery (10); however, postoperative intractable cough remains a neglected clinical issue, for which clinical features and causes are still unclear.

Postoperative refractory cough can occur after all general thoracic operations, such as pulmonary wedge resection, segmentectomy, lobectomy, esophagectomy, and even mediastinal surgery. Potential mechanisms of PIC are (I) airway inflammation or airway hyper-responsiveness after pulmonary resection; (II) anatomic and physiological changes of the air ways; (III) foreign body irritation of bronchial stump; and (IV) underlying diseases, such as cough variant asthma, upper airway cough syndrome, or gastroesophageal reflux-induced cough. The reported risk factors include female sex, history of COPD, obstructed airways, anesthesia duration, right lung cancer, mediastinal lymph node dissections, etc. $(11,12)$. In our present study, we included only lung cancer patients who underwent right upper lobectomy and systematic lymph node dissections and found that the bronchial morphological changes, such as RUL bronchial stump length, bronchial kink, and diameter change of RLL basal bronchus, were independent predictors of PIC.

Bronchial detachment is an important part of lobectomy. It is widely accepted that the management of bronchial stump during surgery has an effect on the occurrence of PIC. Possible causes of stump-related PIC are local inflammatory reactions caused by injury of lung tissue, surgical scar of the airway mucosa, and stimulation by a foreign body, such as sutures or clips in the bronchi (13-15). In our present study, we found that bronchial stump length was an independent risk factor for PIC. However, it is unclear why suturing of the stapled stumps with Prolene was found not be associated with postoperative cough. This might be related to the high biocompatibility of titanium alloy and the nonabsorbable Prolene suture.

Surgeons are well aware of the occurrence of bronchial stenosis and deformation after lobectomy (16). It is regarded as a physiological outcome in most instances; however, unfavorable postoperative complications are occasionally associated with these changes. Limited studies have focused on this issue, especially in relation to postoperative cough. Ueda et al. first reported the clinical ramifications of bronchial kink after upper lobectomy in 50 patients (7). For $42 \%(21 / 50)$ of patients, a bronchial kink was confirmed, and significantly more patients complained of intractable cough and shortness of breath in the kink group compared to the nonkink group (76\% vs. 21\%). Arai et al. reviewed 100 lung cancer patients who underwent right upper lobectomy and found 23 cases with intermediate bronchial kinking after surgery. They concluded that intermediate bronchial kink correlated with postoperative respiratory symptoms (17). In our study, significant changes in bronchial morphology were demonstrated after lobectomy. As shown in Table 2, the diameters of bronchi and angles between different bronchi decreased significantly, which indicates that the remaining airway and lung tissues underwent a process of readjustment after upper lobectomy. Bronchial kink was verified in 27 $(24.1 \%)$ cases, which is consistent with previous reports $(7,17)$. Furthermore, we found that bronchial kink was an 
independent predictor of PIC, which was hypothesized but not proven with clinical data in the literature. Bronchial kink was reported to be associated with reduced functional lung volume and ventilatory capacity, and these may be related to one and the same cause (18). Bronchial stenting has been reported to treat extreme bronchial kinks that cause dyspnea and cough due to bronchial obstruction or atelectasis $(19,20)$. Symptoms of patients were shown to be alleviated after the stenting procedure according to case reports $(19,20)$. Gu et al. investigated the structural and functional alterations of the airways after left upper lobectomy. They reported that the left lower bronchus lumens reduced in most cases by $15 \%$ to $75 \%$, and that wall pressure, airflow velocity, and pressure drop increased significantly postoperatively as measured by the virtual computational fluid dynamics method (21). Therefore, it is assumed that high-speed airflow caused by bronchial kink mechanically stimulates the rapid adapting receptors (RARs) of the major airway tract, passing through the A delta $(\mathrm{A}-\delta)$ fiber to the cough center of the brain, thus inducing coughing. To dilate and correct the deformed airway medically, we attempted to use bronchodilators as an inhalation drug when the common antitussives were ineffective. Our result was consistent with that of Sawada et al. reported in 2012 (22). For a large proportion of PIC patients, symptoms were alleviated after $\beta$-agonist nebulization drugs were administered. The underlying mechanisms were likely the anti-inflammatory effect of the corticosteroid (decreasing the release of certain chemical substances, such as bradykinin, histamine or prostaglandin, and weakening the C-fiber conduction) and the bronchial relaxation effect of a $\beta$-agonist (weakening the A- $\delta$ fiber conduction) (22).

As described above, for the management of PIC, medical treatments to control symptoms are still the approach of choice. Surgical treatment mainly focuses on intraoperative prevention and improvement of surgical manipulations. Surgeons should make sure the operation is conducted with care, protecting nerves and avoiding excessive traction of tissues. The only specific preventive method is the surgical fat-filling procedure after mediastinal lymphadenectomy, as reported by Huang et al. (23). Their theory is based on RARs, which are the main receptors mediating cough and which are mainly located just below the carina and around the main bronchi (24). Huang et al. proposed filling the postlymphadenectomy residual cavities with mediastinal fat tissue or a gelatin sponge to reduce chemical and mechanical stimulation after surgery. In our case, we performed systematic lymphadenectomy for all lung cancer patients and used a medical sponge to fill the cavities to reduce the incidence of PIC. Another surgical procedure that might play a role in the management of PIC is dissecting the inferior pulmonary ligament (IPL) (25). Previous studies reported that dividing IPL might have an adverse effect on bronchial distortion and pulmonary function $(26,27)$. Although no direct correlation between dissecting IPL and PIC has been demonstrated in the literature, bronchial deformation and worsening lung function are potential causes of dry cough after surgery. One systematic review indicated that no clear evidence is available that dissection with IPL can improve clinical outcomes and reduce complications during upper lobectomy (28); consequently, dissection of IPL is not recommended as routine procedure from the point of view of reducing postoperative cough.

Our study has several limitations. First, our study was retrospective in design, and thus there was no quantitative evaluation of patients' coughs. Second, several clinical features of the cohort, such as some surgical details and postoperative pulmonary function data, were not captured, as they were not included in the medical record database. In addition, although the data on bronchial morphology were measured by 2 authors independently based on 3D reconstructive imaging, manual manipulation might have introduced human error. The relatively small sample and the single-center study design warrant further verification in multicenter, prospective, largescale studies both in relation to the study of risk factors of PIC and the potential benefit of nebulization drugs.

\section{Conclusions}

After right upper lobectomy, the morphology of the remaining bronchial tree in the residual lung changed significantly. Bronchial morphological alternations, such as RUL stump length, bronchial kink, and diameter change of the RLL basal bronchus were independent risk factors of postoperative refractory cough. The identification of risk factors associated with PIC can provide surgeons with new ideas for improving the quality of life of lung cancer patients after pulmonary resection.

\section{Acknowledgments}

Funding: None.

\section{Footnote}

Conflicts of Interest: All authors have completed the ICMJE 
uniform disclosure form (available at https://dx.doi. org/10.21037/qims-21-368). The authors have no conflicts of interest to declare.

Ethical Statement: The authors are accountable for all aspects of the work in ensuring that questions related to the accuracy or integrity of any part of the work are appropriately investigated and resolved. The study was conducted in accordance with the Declaration of Helsinki (as revised in 2013). This study was approved by the Renmin Hospital of Wuhan University Review Board for Clinical Research. Informed consent was waived due to the retrospective nature of this study.

Open Access Statement: This is an Open Access article distributed in accordance with the Creative Commons Attribution-NonCommercial-NoDerivs 4.0 International License (CC BY-NC-ND 4.0), which permits the noncommercial replication and distribution of the article with the strict proviso that no changes or edits are made and the original work is properly cited (including links to both the formal publication through the relevant DOI and the license). See: https://creativecommons.org/licenses/by-nc-nd/4.0/.

\section{References}

1. Wang L, Li T, Hong J, Zhang M, Ouyang M, Zheng X, Tang K. 18F-FDG PET-based radiomics model for predicting occult lymph node metastasis in clinical N0 solid lung adenocarcinoma. Quant Imaging Med Surg 2021;11:215-25.

2. Sawabata N, Maeda $H$, Takeda S, Inoue $M$, Koma $M$, Tokunaga T, Matsuda H. Persistent cough following pulmonary resection: observational and empiric study of possible causes. Ann Thorac Surg 2005;79:289-93.

3. Zhu YF, Wu SB, Zhou MQ, Xie MR, Xiong R, Xu SB, $\mathrm{Xu}$ GW. Increased expression of TRPV1 in patients with acute or chronic cough after lung cancer surgery. Thorac Cancer 2019;10:988-91.

4. Pan LY, Peng LP, Xu C, Ding C, Chen J, Wang WY, Zhu XY, Zhao J, Li C. Predictive factors of cough after uniportal video-assisted thoracoscopic pulmonary resection. J Thorac Dis 2020;12:5958-69.

5. Kadokura M. Bronchial deformation associated with lobectomy. Ann Thorac Cardiovasc Surg 2015;21:301-4.

6. Matsuoka H, Nakamura H, Nishio W, Sakamoto T, Harada H, Tsubota N. Division of the pulmonary ligament after upper lobectomy is less effective for the obliteration of dead space than leaving it intact. Surg Today 2004;34:498-500.

7. Ueda K, Tanaka T, Hayashi M, Tanaka N, Li TS, Hamano K. Clinical ramifications of bronchial kink after upper lobectomy. Ann Thorac Surg 2012;93:259-65.

8. Singh R, Kalra MK, Homayounieh F, Nitiwarangkul C, McDermott S, Little BP, Lennes IT, Shepard JO, Digumarthy SR. Artificial intelligence-based vessel suppression for detection of sub-solid nodules in lung cancer screening computed tomography. Quant Imaging Med Surg 2021;11:1134-43.

9. Gao K, Yu PM, Su JH, He CQ, Liu LX, Zhou YB, Pu $\mathrm{Q}$, Che GW. Cardiopulmonary exercise testing screening and pre-operative pulmonary rehabilitation reduce postoperative complications and improve fast-track recovery after lung cancer surgery: A study for 342 cases. Thorac Cancer 2015;6:443-9.

10. Loop T. Does thoracic epidural anaesthesia constitute over-instrumentation in video- and robotic-assisted thoracoscopic lung parenchyma resections? Curr Opin Anaesthesiol 2021;34:199-203.

11. Lin R, Che G. Risk factors of cough in non-small cell lung cancer patients after video-assisted thoracoscopic surgery. J Thorac Dis 2018;10:5368-75.

12. Xie MR, Zhu YF, Zhou MQ, Wu SB, Xu GW, Xu SB, Xu $M Q$. Analysis of factors related to chronic cough after lung cancer surgery. Thorac Cancer 2019;10:898-903.

13. Leuzzi G, Alessandrini G, Forcella D, Facciolo F. Expectoration of the staple line: a delayed complication after previous lobectomy. Interact Cardiovasc Thorac Surg 2015;20:672-4.

14. Di Crescenzo V, Laperuta P, Napolitano F, Carlomagno C, Danzi M, Amato B, Garzi A, Vitale M. Migration of surgical clips through a right lobectomy stump mimicking an asthmatic syndrome. BMC Surg 2013;13 Suppl 2:S32 .

15. Garcia-Olivé I, Andreo F, Rosiñol O, Sanz-Santos J, Font A, Monsó E. Bronchial stump aspergillosis after lobectomy for lung cancer as an unusual cause of false positive fluorodeoxyglucose positron emission tomography and computed tomography: a case report. J Med Case Rep 2011;5:72.

16. Nonaka M, Kadokura M, Tanio N, Yamamoto S, Kataoka $\mathrm{D}$, Inoue $\mathrm{K}$, Takaba T. Changes in lung lobar volume and bronchial deformity after right upper lobectomy. Surg Today 1998;28:285-8.

17. Arai H, Tajiri M, Masuda H, Sekine A, Okudela K, Komatsu S, Iwasawa T, Masuda M. Intermediate bronchial kinking after right upper lobectomy for lung cancer. Asian 
Cardiovasc Thorac Ann 2021;29:19-25.

18. Seok Y, Cho S, Lee JY, Yang HC, Kim K, Jheon S. The effect of postoperative change in bronchial angle on postoperative pulmonary function after upper lobectomy in lung cancer patients. Interact Cardiovasc Thorac Surg 2014;18:183-8.

19. Van Leuven M, Clayman JA, Snow N. Bronchial obstruction after upper lobectomy: kinked bronchus relieved by stenting. Ann Thorac Surg 1999;68:235-7.

20. Hutchinson CT, DiBardino D, Ma K, Jarrar D, Singhal S, Haas A. Airway Stenting for Treatment of Left Lower Lobe Airway Obstruction after Left Upper Lobectomy. Ann Am Thorac Soc 2019;16:1444-6.

21. Gu Q, Qi S, Yue Y, Shen J, Zhang B, Sun W, Qian W, Islam MS, Saha SC, Wu J. Structural and functional alterations of the tracheobronchial tree after left upper pulmonary lobectomy for lung cancer. Biomed Eng Online 2019;18:105.

22. Sawada S, Suehisa H, Yamashita M. Inhalation of corticosteroid and $\beta$-agonist for persistent cough following pulmonary resection. Gen Thorac Cardiovasc Surg 2012;60:285-8.

23. Huang J, Luo Q, Tan Q, Lin H, Qian L, Ding Z.

Cite this article as: Lu XF, Min XP, Lu B, Fan GH, Zhu TY. Bronchial morphological changes are associated with postoperative intractable cough after right upper lobectomy in lung cancer patients. Quant Imaging Med Surg 2022;12(1):196206. doi: 10.21037/qims-21-368
Evaluation of the surgical fat-filling procedure in the treatment of refractory cough after systematic mediastinal lymphadenectomy in patients with right lung cancer. J Surg Res 2014;187:490-5.

24. Widdicombe J. Reflexes from the lungs and airways: historical perspective. J Appl Physiol (1985) 2006;101:628-34.

25. Usuda K, Sagawa M, Aikawa H, Tanaka M, Machida Y, Ueno M, Sakuma T. Do Japanese thoracic surgeons think that dissection of the pulmonary ligament is necessary after an upper lobectomy? Surg Today 2010;40:1097-9.

26. Seok Y, Yi E, Cho S, Jheon S, Kim K. Perioperative outcomes of upper lobectomy according to preservation or division of the inferior pulmonary ligament. J Thorac Dis 2015;7:2033-40.

27. Bu L, Yang AR, Peng H, Xu ZY, Wu JQ, Wang P. Dividing inferior pulmonary ligament may change the bronchial angle. J Surg Res 2016;201:208-12.

28. Khanbhai M, Dunning J, Yap KH, Rammohan KS. Dissection of the pulmonary ligament during upper lobectomy: is it necessary? Interact Cardiovasc Thorac Surg 2013;17:403-6. 